\section{Cureus}

\title{
Binding of NRSF to the HCN1 Gene Represses Expression after Seizures
}

\author{
Romela Petrosyan $^{1}$ \\ 1. University of Central Florida College of Medicine
}

$\square$ Corresponding author: Romela Petrosyan, romela.petrosyan@knights.ucf.edu Disclosures can be found in Additional Information at the end of the article

\section{Abstract}

Epilepsy is a devastating disorder that affects millions of people worldwide. One of the most common types of epilepsy, temporal lobe epilepsy (TLE), is associated with significant morbidity in cognitive and psychosocial dysfunction. TLE has long been correlated with a history of prolonged febrile seizures in childhood; hence, understanding the consequences of prolonged febrile seizures on TLE is of considerable clinical significance. The Baram Laboratory has consistently observed down-regulation after seizures of the hyperpolarization-activated cyclic-nucleotide gated 1 (HCN1) channels that are responsible for mediating hyperpolarization-activated (Ih) currents. In the hippocampus, these currents regulate the resting membrane potential, shape rhythmic and synchronized neuronal activity, and regulate the temporal summation of dendritic depolarization. This study targets the factors responsible for regulating the transcription of the HCN1 gene. By using chromatin immunoprecipitation (ChIP), polymerase chain reaction (PCR), and DNA electrophoresis, we show that the neuron restrictive silencer factor (NRSF) binds to the NRSE region in the first intron of the HCN1 gene. NRSF, in conjunction with its cofactors, deacetylates and methylates the chromatin, preventing transcription of the HCN1 gene. Such biochemical change may produce neuronal injury to the developing brain, promoting the onset of epilepsy.

Categories: Genetics, Internal Medicine, Neurology

Keywords: gene expression, temporal lobe epilepsy, neuron restrictive silencer factor (nrsf), hyperpolarization activated cyclic-nucleotide gated 1 (hcn1), epilepsy

\section{Introduction}

Febrile seizures are convulsions that are caused by fever exceeding $102^{\circ} \mathrm{F}\left(39^{\circ} \mathrm{C}\right)$ in children up to five years of age. Although they are mostly harmless due to their short durations and small number of reoccurrences, there is a small percentage of complex febrile seizures-prolonged and reoccurring seizures-that may cause a number of significant changes to occur in an infant brain. Many correlation studies have established a relationship between individuals who have experienced complex febrile seizures and temporal lobe epilepsy (TLE), one of the most common types of epilepsy. Nevertheless, according to Brewster, et al., 2000, "the direct causal relationship of prolonged febrile seizures to this common epilepsy, and the mechanisms by which these early life seizures might be proepileptogenic, have not been resolved" [1]. Although much remains unknown, a study using a rat model demonstrated functional changes in the hippocampus after complex febrile seizures [2].

Whole-cell patch clamp recordings from the hippocampal CA-1 pyramidal cells have demonstrated an alteration in the biophysical properties of the Ih current by experimental febrile seizures [3]. The Ih current is mediated by the hyperpolarization-activated cyclic 
nucleotide-gated (HCN) channels, some of the most important regulatory proteins in the hippocampus [4]. Out of the four isoforms, HCN1 channels are dominant in hippocampal pyramidal cells and CA1 interneurons under normal conditions [5-6]. The Baram laboratory previously observed a reduction in HCN1 levels after febrile seizures [1], and the main objective of our study is to present the reason behind such a decrease in HCN1 expression.

Many neuronal and non-neuronal genes contain a DNA sequence element, known as the neuron restrictive silencer element (NRSE). The neuron restrictive silencer factor (NRSF) binds to the NRSE sequence and represses neuronal gene transcription [7]. Since the HCN1 gene contains an NRSE sequence in its first intron, we hypothesized that the reduction of the number of HCN1 channels might be due to the binding of the NRSF to the NRSE region in the HCN1 gene, preventing it from being transcribed. A rodent model was employed because the NRSE sequence in the HCN1 gene and the structure of the NRSF gene are conserved in humans, mice, and rats [8], as shown in Figure 1.

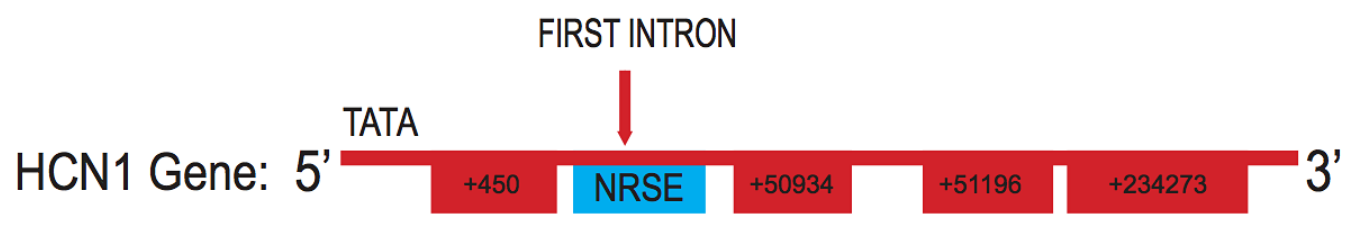

Alignment of HCN1 genes for Intron 1 NRSE: Consensus NRSE: ttCAGCACCa Reverse Consensus NRSE: GgCgCTgTCC CGGACAGcgC C $\star \star-* \star \star \star \star * \star *$ gtGGTGCTGa a

HCN1 Human:

1159

GGAGCTGTCC $---* *-* * * *$ a HCN1 Mouse:

\section{FIGURE 1: HCN1 Gene Contains a Conserved NRSE}

HCN1 Gene Contains a Conserved NRSE (McClelland, et al., 2008)

Aside from the NRSE consensus sequence (the series of nucleotides most frequently found at each position within a region of DNA), the model is constructed to match the stage of brain development at 18 months [10], when an infant brain is most susceptible to experiencing febrile seizures. An 18-month human brain is equivalent in its extent of maturation to a brain of a postnatal 10 (P10) rat [11]. The pups are injected with kainic acid, a seizure-inducing neurotoxin, to induce status epilepticus [1]. Forty-eight hours after injection, their hippocampi are resected to analyze NRSF binding. For the HCN1 gene to be expressed, the tightly packed DNA must be unwound to allow the cell's transcription machinery to transfer the genetic information from DNA in the form of messenger RNA, which is subsequently used in manufacturing the HCN1 channel. However, by binding to the NRSE region in the first intron of the HCN1 gene, NRSF allows subsequent recruitment of its cofactors that stimulate the methylation (chemical modification via attachment of $\mathrm{CH} 3$ groups) of the nearby histones (DNA-packaging proteins), preventing them from unwinding to allow DNA transcription, as illustrated in Figure 2. This phenomenon is observed directly through chromatin immunoprecipitations (ChM) by targeting the NRSF binding factors believed to be involved in the repression of the HCN1 expression. Thus, dimethyl histone and NRSF antibodies are used 


\section{Cureus}

to immunoprecipitate DNA to which the modified histone or NRSF has bound, respectively. At the completion of ChIP, the extracted DNA from the control and seizure-induced hippocampi is replicated using polymerase chain reaction (PCR) with specific primers for the HCN1 gene and the actin gene. Mainly used for comparison purposes because it does not contain an NRSE sequence, the actin gene is highly conserved across species, common, and highly expressed. Since NRSF is overexpressed after seizures, it is expected that the quantity of the recovered DNA will be higher in rats injected with kainic acid, a convulsant, than those that were not injected due to increased NRSF binding to the HCN1 gene in rats that have experienced seizures.



FIGURE 2: NRSF Binding Mechanism

NRSF Binding Mechanism (McClelland, et al., 2008)

\section{Materials And Methods}

All experiments were carried out in accordance with the Institutional Animal Care and Use Committee at the University of California, Irvine and were consistent with federal guidelines. Charles-River delivered pregnant rats (Embryonic 16) that each gave birth to a litter of 8-10 pups four to five days later (Embryonic 20-21). Different litters were divided into littermate pairs in which one animal was assigned to the control group, while the other was placed into the experimental group. The pups were allowed to mature to 10 days (P10) before the experimental group was injected with kainic acid, a seizure-inducing toxin. Forty-eight hours after the injection, the hippocampi of each littermate set were resected and stored at $-80^{\circ} \mathrm{C}$.

\section{Chromatin immunoprecipitation}

To avoid degradation and other undesirable reactions, all of the samples and their additives were kept on ice. 
Day 1: Frozen hippocampi were fixed by adding $1 \mathrm{~mL}$ of cold $1 \%$ paraformaldehyde (PFA) (Sigma Aldrich; prepared by dissolving $0.05 \mathrm{~g}$ of powdered PFA in $5 \mathrm{~mL}$ of PBS and rotating for at least $120 \mathrm{~min}$ ) in phosphate buffered saline (PBS; 10x stock: $2.28 \mathrm{~g}$ monobasic NaH2PO4, 11.5g dibasic Na2HPO4, and 43.84g NaC1 dissolved in 450mL deionized water, adjusted $\mathrm{pH}$ to 7.4 with $1 \mathrm{M} \mathrm{HC1}$, and brought the volume to $500 \mathrm{~mL}$ with water; diluted to $1 \mathrm{x}$ before use) and incubating for five minutes on a rotator at $4^{\circ} \mathrm{C}$. The samples were then spun at $1000 \mathrm{rpm}$ for five minutes, the supernatant was discarded, and the hippocampi were washed twice with an inhibitor solution of $20 \mu \mathrm{L}$ of 50xPIC (Protease Inhibitor Cocktail; Roche; pellet diluted in deionized water) in $1 \mathrm{~mL}$ of $0.125 \mathrm{M}$ glycine in PBS O.T. Baker).

The hippocampi were then lysed with $360 \mu \mathrm{L}$ of radio immuno precipitation assay (RIPA) lysis buffer (prepared by mixing $0.15 \mathrm{M} \mathrm{NaC1}, 5 \mathrm{Mm}$ Ethylenediamine Tetraacetic Acid (EDTA)/pH 8, 1\% Triton x100, and 10 m.M Trizma hydrochloride/pH 7.4 all from Boston Bioproducts; just before using, added: 1:1000 5 M DTT, 1:1000 100 mM PMSF in isopropanol, and 1:1000 $5 \mathrm{M}$ aminocaproic acid) and $8 \mu \mathrm{L}$ of 50xPIC and sonicated at $20 \%$ for $20 \mathrm{sec}$ ( $1 \mathrm{sec}$ on $/ 5 \mathrm{sec}$ off $)$ with a Branson Digital Sonifier 450 (VWR Scientific). Immediately after sonication, $20 \mu \mathrm{L}$ of 50xPIC (Protease Inhibitor Cocktail) was added and the broken hippocampi centrifuged at $13.2 \mathrm{krpm}$ for five minutes and $350 \mu \mathrm{L}$ of the supernatant transferred to new tubes. After adding $1 \mathrm{~mL}$ of RIPA (Radio Immuno Precipitation Assay) lysis buffer, $425 \mu \mathrm{L}$ of the supernatant was relocated to new tubes. To target the factors responsible for the decrease of the HCN1 channels, either dimethyl histone antibody (3p.g), immunoglobulin G (IgG) antibody (3 p.g), or H290 NRSF antibody (3 p.g) was added to each sample. IgG served as a negative control that does not bind to any proteins and was used to immunoprecipitate non-specific DNA.

Day 2: Thirty $\mu \mathrm{L}$ of Protein G Agarose Beads/Salmon Sperm DNA (Upstate/Millipore) were obtained per immunoprecipitation (IP) sample distributed in new $1.6 \mathrm{~mL}$ tubes. The Protein G Agarose Beads were spun at 6000 rpm for 30 seconds, and the supernatant was aspirated. Initially, the beads were washed three times by adding $1 \mathrm{~mL}$ of RIPA (Radio Immuno Precipitation Assay) lysis buffer, spinning the tube at $6000 \mathrm{rpm}$ for 30 seconds, and aspirating the supernatant. At the end of the last wash, $1 \mathrm{~mL}$ of RIPA lysis buffer was added to the washed Protein G Agarose Beads and $90 \mu \mathrm{L}$ was distributed into new tubes. Meanwhile, the IP samples were spun at $13.2 \mathrm{krpm}$ for $10 \mathrm{~min}$ and $360 \mathrm{pLL}$ of the supernatant transferred to the washed Protein G Agarose Beads. The samples incubated on a rotator for $60 \mathrm{~min}$ at $4^{\circ} \mathrm{C}$. After incubation, they were centrifuged at $6000 \mathrm{rpm}$ for one minute, and the supernatant removed, followed by five washes. During each wash, $1 \mathrm{~mL}$ of RIPA lysis buffer was added, the samples were incubated at $4^{\circ} \mathrm{C}$ for $5 \mathrm{~min}$, spun at $6000 \mathrm{rpm}$ for one minute, and the supernatant was discarded.

The DNA was purified by adding $65 \mu \mathrm{L}$ of $20 \%$ Chelex 100 (made in deionized water; Bio-Rad) to each sample, vortexing the samples for five seconds and locking them with clasps before incubating them in a boiling water bath for 10 minutes. The inputs (additional leftovers of DNA) underwent the same procedure but received only $10 \mu \mathrm{L}$ of $20 \%$ Chelex. Finally, the samples were centrifuged at $13.2 \mathrm{krpm}$ for one minute at $4^{\circ} \mathrm{C}$ and $35 \mu \mathrm{L}$ of the supernatant was transferred into new $0.6 \mathrm{~mL}$ tubes for storage at $-80^{\circ} \mathrm{C}$.

\section{Polymerase chain reaction}

To equilibrate the quantity of DNA in the subsequent samples, the DNA from the inputs was amplified and quantified using the PCR and then separated in DNA electrophoresis. The samples were prepared by mixing nucleotide free water (Promega; volume varied with respect to concentration), $1 \mu \mathrm{L}$ of Primers (Forward primer in the first intron of the HCN1 gene: AGGGAGCTGTCCACAGTTCTGAAT/ Reverse primer in the 1st intron of the HCN1 gene: AGATGGACGGTCATCAAACC; Forward primer in the 3rd exon of the actin gene: GACTACCTCATGAAGATCCTGACC/Reverse primer in the 3rd exon of the actin gene: 
GAGACTACAACTTACC CAGGAAGG) at a 2:1:1 ratio-actin (0.625 p.M), HCN1 (0.3125 p.M), DNA template (volume varied with respect to concentration), and $10 \mu \mathrm{L}$ of Gotaq Green Master Mix (Promega), in that order. The total volume of all samples was $20 \mu \mathrm{L}$. The samples were placed into the Eppendorf Mastercycler Gradient-Thermocycler for 33-36 cycles. The number of cycles was determined by trial and error to obtain clear, differentiable bands for analysis.

\section{DNA electrophoresis}

DNA electrophoresis was done with a 3\% large gel, prepared by dissolving $2.1 \mathrm{~g}$ of Agarose (Apex) in $70 \mathrm{~mL}$ of TAE buffer (50x stock: $242 \mathrm{~g}$ Tris base (Sigma) in $750 \mathrm{~mL}$ deionized water, $57.1 \mathrm{~mL}$ Acetic Acid (Fisher Biotech), $100 \mathrm{~mL}$ of $0.5 \mathrm{M}$ Ethylenediamine Tetraacetic Acid (EDTA/pH 8.0; Boston Bioproducts), diluted to 1x before use), and adding $40 \mu \mathrm{L}$ of Ethidium Bromide. The mixture was microwaved at 20-, 15-, and 6-second intervals for a total of approximately 91 seconds to obtain a clear, viscous solution free of air bubbles that might disrupt the clear visualization of the gel. At the completion of the PCR, the samples $(15 \mu \mathrm{L})$ and Bioline Hyperladder I ( $5 \mu \mathrm{L}$ and $2.5 \mu \mathrm{L}$ in the first and last wells; Bioline) were loaded into their appropriate wells, and the gel was run for $5 \mathrm{~min}$ at $157 \mathrm{~V}$ and 1 hour at $125 \mathrm{~V}$.

The gel was visualized under UV light at 302nm and photographed with a digital camera. The image was reproduced in P.S. Remote (version 1.5.8) and saved for further analysis with Image J (Image Processing and Analysis in Java). After outlining the bands and Hyperladder I, the program produced peaks that corresponded to the bands seen on the gel. Greater band intensity indicated more of the immuno-precipitated DNA and larger peak areas. For comparison purposes, an optimal linear range was obtained by running the PCR of the recovered DNA using different DNA concentrations and reaction cycle numbers. Moreover, duplicates of each DNA amount and reaction number were performed. A quantitative DNA ladder was used in DNA electrophoresis to produce a standard curve of band intensities vs. DNA amounts. From each littermate pair, the quantities of the recovered DNA of the targeted genes in the kainateinduced rats were divided by the amount of recovered DNA from the control rats to yield ratios, indicating the change in binding to the HCN1 and actin gene.

\section{Results}

The use of ChIP, PCR, and DNA electrophoresis permitted a direct observation of the neuronal changes in the developing brain that are experienced after seizure. Analyses of the gels after DNA electrophoresis have revealed bands with higher intensity, indicating higher DNA concentrations in rats injected with kainic acid than those that were not. The ratios comparing binding to the HCN1 and actin gene are shown for ChIP assays performed with an NRSF antibody and dimethylated histone antibody. A higher amount of recovered DNA implies higher antibody binding during ChIP, which indicates greater NRSF binding to the gene in rats injected with kainic acid. When NRSF binds to the first intron of the HCN1 gene, it blocks access for the cellular transcription machinery to produce the primary mRNA for the HCN1 protein. Overall, normal HCN1 expression exists before seizures. However, NRSF levels are upregulated after kainic acid-induced seizures [12], leading to increased NRSF binding to the HCN1-NRSE region. This changes chromatin reception to transcription and lowers HCN1 expression, as seen in Figure 3. Moreover, binding of NRSF to the NRSE region promotes methylation of the histones around the gene itself. Chromatin is more receptive to transcription in the acetylated form than in the methylated form due to the tight packing of the histones in the methylated state. Therefore, transcription of the HCN1 gene is repressed (Figure 4). 


\section{Cureus}

ChIP DNA recovered in SE vs Controls

NRSF Antibody

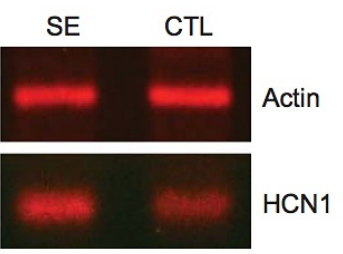

ChIP DNA recovered and multiplied using PCR. Representative photograph of PCR products run using agarose gel electrophoresis.

$\mathrm{SE}=$ seizure $\quad \mathrm{CTL}=$ control

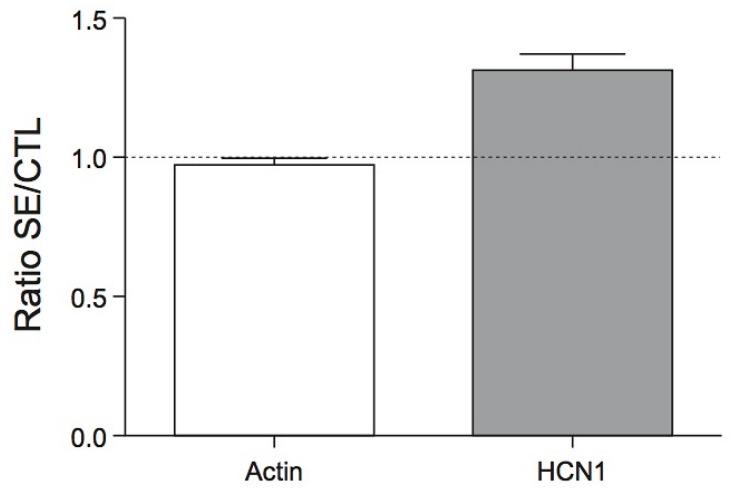

Error Bars indicate SEM. $n=4$ littermate pairs.

\section{FIGURE 3: Data Visualizations}

Higher DNA recovery with NRSF antibody in kainate-induced-seizure rats than in control rats for HCN1 but no difference for Actin.

\section{Dimethylated H3K9 Histone Antibody}

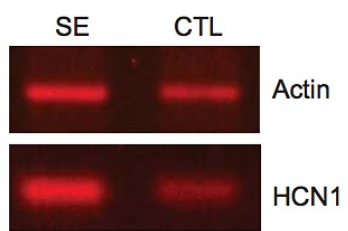

ChIP DNA recovered and multiplied using PCR. Representative photograph of $\mathrm{PCR}$ products run using agarose gel electrophoresis.

$\mathrm{SE}=$ seizure $\quad \mathrm{CTL}=$ contro
ChIP DNA recovered in SE vs Controls

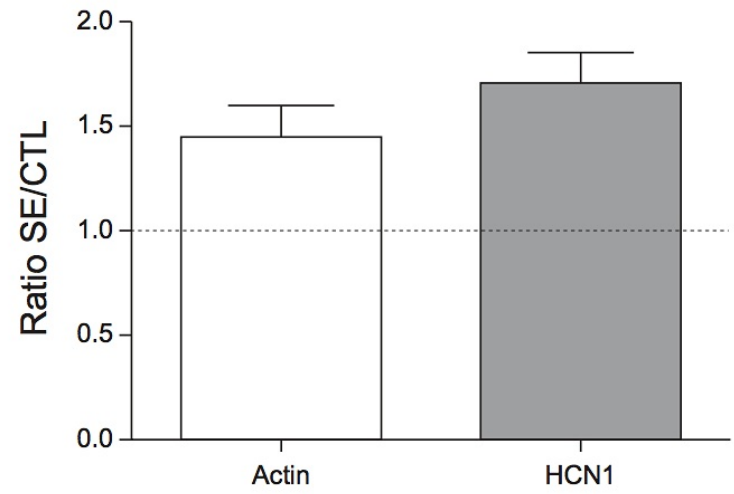

Error Bars indicate SEM. $n=5$ littermate pairs.

\section{FIGURE 4: Data Visualizations}

The HCN1 gene environment is more methylated after kainic acid.

\section{Discussion}

This study demonstrates that HCN1 expression is repressed after seizures due to the binding of the NRSE. In a ChIP assay using the NRSF antibody, the seizure/control ratio of 1 in actin implies no difference in the level of expression of the actin protein in either seizure-induced or control rats. However, a higher than $1 \mathrm{HCN} 1$ ratio is indicative of higher immunoprecipitation of DNA due to higher NRSF binding to the NRSE region in the HCN1 gene in seizure-induced rats than in control rats. Hence, HCN1 channels are downregulated in seizure-induced rats. On the other hand, the ChIP assay using a dimethylated histone antibody has a higher HCN1 ratio, indicating increased methylation of histones near the HCN1 gene in that suppress its 
expression versus control rats. The actin ratio is lower than the HCN1 ratio because of lower deviation in actin expression in seizure-induced rats compared to control rats. However, the actin ratio of above one is due to the regulation of its expressivity by external factors not addressed in this study. Such observations serve as significant discoveries in understanding the mechanism of epileptogenesis.

While the animal model is a good approximation of human infant seizures, it holds a significant bias in attempting to simulate human conditions. Neuronal changes observed in a homogeneous rodent population cannot be applied to humans because the human population is very far from being characterized as homogeneous. Dr. Celine M. Dube, et al. furthers this issue by stating the following:

"Whether epileptogenic processes occurring in the immature rodent brain are analogous directly to those in children remain largely unknown......[However], this issue will be resolved by interventional studies using molecular targets discovered in the animal model and by aiming to prevent human epileptogenesis" [13].

\section{Conclusions}

Molecular research such as this holds great potential for understanding the mechanisms of epilepsy development, which may inspire design of future preventative and interventional approaches. Although HCN1 levels are reduced with respect to the increased NRSF levels after seizures, we are currently monitoring the persistence of these changes at various time intervals after the seizure. Moreover, a possible prevention method is being tested that aims to reduce NRSF binding after seizures with the use of decoy oligonucleotides, short DNA sequences of the NRSE.

\section{Additional Information}

\section{Disclosures}

Human subjects: All authors have confirmed that this study did not involve human participants or tissue. Animal subjects: The Institutional Animal Care and Use Committee at the University of California, Irvine Issued protocol number N/A. Conflicts of interest: In compliance with the ICMJE uniform disclosure form, all authors declare the following: Payment/services info: This work was supported by NIH R37 N535439. Financial relationships: All authors have declared that they have no financial relationships at present or within the previous three years with any organizations that might have an interest in the submitted work. Other relationships: All authors have declared that there are no other relationships or activities that could appear to have influenced the submitted work.

\section{Acknowledgements}

About the Author: Having a sister who has struggled with seizures since the age of six months, Romela Petrosyan hoped to find a research group where she could gain more insight about her sister's disorder and was ecstatic to be accepted into Dr. Baram's laboratory. The ultimate goal of the lab is to build a clear understanding of the mechanism of epileptogenesis from febrile seizures, which will allow for new medical means to counter the development of epilepsy. Romela is thrilled to be a part of discovering ways to help the countless people who suffer from this disorder, and she plans to continue her education through medical school, eventually becoming a physician. Faculty Mentor: Tallie Z. Baram "Epilepsy is the most common brain disorder of young people, but the mechanisms that convert a normal brain into an epileptic one are unknown. Sometimes, infection or injury can kill brain cells or damage them; however, when epilepsy begins early in life, there is often no evidence of injury. Romela examined the possibility that in response to specific insults, there are "master switches" that attach to genes 
within neurons and change their expression, modifying how neurons act. She focused on NRSF and found a massive increase in its binding to DNA of several important neuronal genes, leading to loss of proteins encoded by these genes and perturbing the ability of neurons to communicate. Romela's project provides a mechanism for the conversion of a normal brain into a diseased one, and hopefully points toward a means of reversing the process.”

Acknowledgements: This work was supported by NIH R37 N535439. I am greatly indebted to my supervisor, Shawn McClelland, for his consistent support and assistance throughout this project, Dr. Tallie Z. Baram for her insightful wit that has encouraged me to look at the core of epileptic research, and my parents, without whose love and support I could not have been able to pursue the medical field that I respect and honor.

\section{References}

1. Brewster A, Bender RA, Chen Y, Dube C, Eghbal-Ahmadi M, Baram TZ: Developmental febrile seizures modulate hippocampal gene expression of hyperpolarization-activated channels in an isoform- and cell-specific manner. J Neurosci. 2002, 22:4591-4599.

2. van Gassen KL, Hessel EV, Ramakers GM, et al.: Characterization of febrile seizures and febrile seizure susceptibility in mouse inbred strains. Genes Brain Behav. 2008, 7:578-586.

10.1111/j.1601-183X.2008.00393.x

3. Chen K, Aradi I, Thon N, Eghbal-Ahmadi M, Baram TZ, Soltesz I: Persistently modified hchannels after complex febrile seizures convert the seizure-induced enhancement of inhibition to hyperexcitability. Nat Med. 2001, 7:331-337.

4. Bender RA, Baram TZ: Hyperpolarization activated cyclic-nucleotide gated (HCN) channels in developing neuronal networks. Prog Neurobiol. 2008, 3:129-140.

10.1016/j.pneurobio.2008.09.007

5. Bender RA, Dubé C, Baram TZ: Febrile seizures and mechanisms of epileptogenesis: insights from an animal model. Adv Exp Med Biol. 2004, 548:213-225.

6. Brewster AL, Bernard JA, Gall CM, Baram TZ: Formation of heteromeric hyperpolarizationactivated cyclic nucleotide-gated (HCN) channels in the hippocampus is regulated by developmental seizures. Neurobiol Dis. 2005, 19:200-207.

7. Schoenherr CJ, Anderson DJ: The neuron-restrictive silencer factor (NRSF): a coordinate repressor of multiple neuron-specific genes. Science. 1995, 267:1360-1363.

8. Palm K, Metsis M, Timmusk T: Neuron-specific splicing of zinc finger transcription factor REST/NRSF/XBR is frequent in neuroblastomas and conserved in human, mouse and rat. Brain Res Mol Brain Res. 1999, 72:30-39.

9. McClelland S, Richichi C, Zha Q, Baram TZ: Activity dependent increase of NRSF/ REST levels suppresses expression of the Hyperpolarization Activated Cyclic-Nucleotide Gated (HCN) channel. Proceedings from Society for Neuroscience. 2008, 33.7/D65.

10. Hauser WA: The prevalence and incidence of convulsive disorders in children . Epilepsia. 1994, 35:1-6.

11. Dobbing J: Undernutrition and the developing brain. The relevance of animal models to the human problem. Am J Dis Child. 1970, 120:411-415.

12. Palm K, Belluardo N, Metsis M, Timmusk T: Neuronal expression of zinc finger transcription factor REST/NRSF/XBR gene. J Neurosci. 1998, 18:1280-1286.

13. Dubé CM, Brewster AL, Richichi C, Zha Q, Baram TZ: Fever, febrile seizures and epilepsy. Trends Neurosci. 2007, 30:490-496. 